\title{
Short Term Effects of Kinesotaping with Mobilization on Balance in Patients with Osteoarthritis of Knee Joint
}

\section{Tanushree Ganguly, Md Firoj Alam*, Raghvi Sharma, Nitin Pabla and Taranjot Kaur}

Department of Physiotherapy, Lovely Professional University, Punjab, India

*Corresponding Author: Md Firoj Alam: Department of Physiotherapy, Lovely Professional University, Punjab, India.

Received: July 12, 2019; Published: August 16, 2019

\begin{abstract}
Study Design: Case studies

Background and Purpose: Kinesio taping with Mulligan Mobilization treatment technique is found to be effective on managing pain, improving balance and coordination, Range of Motion for knee joint in osteoarthritic patient. Hence the purpose is to analyse the combination effect of Mulligan's Mobilization with K-Taping on parameters of pain, balance and functional disability with osteoarthritis of kneejoint.
\end{abstract}

Case Description: The 5 patients in this series have clinical evidence of osteoarthritic knee and each underwent a similar treatment program consisting of Kineso-Taping with Mulligan Mobilization with Movement (MWM).

Methods: All the patients were treated with Therapeutic K-Taping followed by Mulligan Mobilization. Outcome measurements used were NPRS for pain, Western Ontario And MC Master Universities Osteoarthritis Index (WOMAC) for Functional disability, Berg Balance Scale (BBS) for balance and coordination and Time Up and Go (TUG) for mobility and static and dynamic balance. The results were measured before intervention and after 48 hours of intervention.

Result: It was found that there is a significant change in mean of NPRS, BERG balance SCORE, TUG SCORE and WOMAC as well as improvement in muscle strength before and after intervention with Kinesiotaping and Mulliganmobilization.

Discussions: All the patients responded well to our program. This case study suggests that patients with clinical evidence of osteoarthritic knee can show a remarkable improvement with Functional disability and static and dynamic balance.

Keywords: Osteoarthritic knee; Kineso Taping; Mulligan Mobilization; Pain; Balance; WOMAC; NPRS; TUG; BERG

\section{Introduction}

Osteoarthritis is a common condition affecting synovial joints. The condition is growing rapidly in India due to altered lifestyles, age factors. There is gender discrimination in OA, studies shows that it is more likely to women than in men. It is found to be more prevalent in females nearly $31 \%$ than in males which is about $28 \%$ [1]. It occurs in elderly persons, nearly $45 \%$ of women over age of 65 have symptoms while radiological evidences are found in $70 \%$ above age of 65 years. The studies shows that prevalence of OA depends on BMI, it is low in underweight people (28\%) as compare to 0 normal or obese persons (33\%). It is highest in people who are obese. Prevalence is higher in people who have a sedentary lifestyle as compared to people who have physically demanding and active lifestyle. Moreover, daily wage workers have lowest prevalence (22\%) (Pulkesh Singh., et al. 2016). Prevalence is higher in people who use western toilets(42\%) as compare to people use Indian toilets(29.7\%). It is more likely to persons who do not exercise(83.9\%)as compare to people who exercise(36\%) [1].

Osteoarthritis causes mild to severe pain due to biomechanical stress on the joints, resulting in difficulties to carry out ADLs [2]. Moreover, it is degeneration caused by progressive loss of articular cartilage and synovial inflammation. Basic pathology of $\mathrm{OA}$ is reduced ability of chondrocytes to respond to growth factors which in turn results in thinning of cartilage, synovial inflammation or osteophyte formation [3]. Symptoms of OA include pain, functional limitation and reduced physical activity which results in muscle wasting around affected joint. Falls are common in OA patients. Studies show that women are more likely prone to falls rather than men. Elderly patients with knee and hip OA adopt various strate- 
gies while walking. They develop Trendelenburg's gait, the tilting of pelvis to opposite side and shift of centre of gravity results in instability. As a result there is poor balance control which results in falls. Studies shows that people with self-reported arthritis had a $50 \%$ increase in risk of recurrent falls. However falls in OA are not always associated with fractures [3].

Kineso tape is an adhesive elastic which is latex free and antiallergic to skin and can be worn for days without any side effect. Kineso taping method is a rehabilitation technique which is designed to enhance the body's natural healing. The taping does not restrict the joint range of motion while providing stability and support to the adjacent muscles. (Zhijun Lu., et al. 2018) Many studies have identified the effect of taping on the musculoskeletal and the nervous system [4].

Manual therapy has proven to be a benefit in management of knee OA. Mulligan Mobilization with Movement is a passive assisted glide performed perpendicularly to joint plain to get rid of positional fault with patient performing to active movement repeatedly with pain levels always reducing post mobilization (Ramya V Rao., et al. 2017).

\section{Review of literature}

Osteoarthritis is slow progressing condition that affects articular cartilage of weight bearing joints [5]. OA of knee joint is known to specifically cause significant pain, stiffness and disability which in effect leads to deteriorating quality of life [5] among the various treatment options available for management OA conservative treatment approaches have been widely explored [6]. Conservative treatment approaches include physiotherapy for knee joint which aims to decrease amount of pain, improve joint function and prevent the progression of condition [6]. However there has been no treatment which outweighs the outcomes of others (Hadamus., et al, 2017).

Kinesio taping has been shown to improve the functional outcome, decrease pain and improve performance in patients with $\mathrm{OA}$ of knee $[7,8]$. The advantage of kinesio taping over other interventions is that it is easy to apply, is cheap and convenient for patient has also shown to improve WOMAC scores and improves quadriceps muscle strength (Sari., et al. 2017)

Mobilization intervention improve extensibility of connective tissue and joint play which helps in decreasing stiffness and pain (French., et al, 2011). MWM works on the principle of connecting faulty patterns of the joint while performing active muscle contraction. When sustained, it connects the postural fault and helps in restoring joint function [9].
In a study by Nam., et al in 2013, Mulligan mobilization given to subjects with $\mathrm{OA}$ knee resulted in significant pain relief and better WOMAC Scores when compared to other conservative treatment approaches. This can be contributed to correction of articular defects and improves articulation as well as pain gait analyze by correction of biomechanically improved joint [10].

Chronic pain patients tend to prefer the NPRS scale over other measures of pain intensity scales, including the pain VAS because of its comprehensibility and ease of completion. However, it focus on group of patients with chronic back pain and symptomatic hip and knee osteoarthritis $(\mathrm{OA})$ have found that the pain in NPRS is not enough in capturing the complexity and varied nature of the pain experience or improvements due to symptom fluctuations. High test-retest reliability has been observed in both literate and illiterate patients with osteoarthritis ( $r=0.96$ and 0.95 , respectively) before and after medical appointment.

NPRS scale is a valid and reliable scale to measure pain intensity. It is superior over the VAS as the ability to be administered both verbally (therefore by telephone) and in writing, as well as its simplicity of scoring. Though, similar to the pain VAS, the pain NPRS evaluates only single component of the pain experience, pain intensity, and therefore does not capture the complexity and idiosyncratic nature of the pain experience or improvements due to symptom fluctuations [11].

The Timed Up and Go (TUG) test is one of the simple and quick tests to assess patient's functional mobility in osteoarthritis patients. TUG test is reliable because it demonstrates good measurement properties in people with OA and other populations. TUG test as a performance-based test of physical function in individuals diagnosed with hip or knee osteoarthritis [12].

WOMAC is a great assessment in osteoarthritis population. It is a disease-specific scale which targeted for pain and difficulty of the knee joint.

One of many advantages of using WOMAC is that it doesn't evaluate upper limb function. The length is important in a postal survey to enhance response rates. The components of WOMAC are pain, stiffness and physical function separately. As it contains 24 questions, that's why it's a bit vast questionnaire.

This contrasts to the HAQ/mHAQ and Lequesne, this reports a single score but this has drawbacks over 24 questionnaire score as they lead to loss of information as thesame score can be obtained from many different compositions of the subdomains of the scale. (Clare Jinks., et al. 2002) 
Berg balance scale is a clinical test for standing and dynamic balance in patient of $\mathrm{OA}$ knee. This test consist of 14 task testing static and dynamic balance of the patients. Each task is scored on a 0 (unable to perform or need assistance) to 4(able to perform independent task or ADL). Scale with maximum score of 56 [13].

\section{Methodology}

An experimental study design with combination of mobilization and kinesio taping was used. A total of five female subjects with osteoarthritis of knee joint with age group 45-60 years were recruited. All the patient were having history of knee osteoarthritis since 2 years to 5 years with Grade 1 to Grade 2 based on radiological finding diagnosed by physician. Subject excluded were osteoarthritis of Hip, any systemic disease, recent fracture of tibia or femur, subject who undergone any lower limb surgery, any types of lower limb deformity, Grade $3 \mathrm{OA}$ and subject taking intra articular cortico steroid. All the subjects fulfilling the inclusion criteria were informed about the research study. Informed consent was taken by the subjects.

The subjects were explained about the study and the procedure to be undertaken. Informed consent from all the subjects was taken. Baseline measurements were taken for muscle strength, pain, balance and coordination. To assess muscle strength, MMT was done for Quadriceps and mstrings bilaterally. For measurement of pain, subjects were asked to fillte NPRS. For balance, coordination and functional balance, TUG and WOMAC scores were filled in respectively. This was followed by treatment intervention with Mulligan mobilization and kinesiotaping.

Mulligan's Movement with Mobilization (MWM) with sustained manual glide of tibia (anterio-posterior and posterio-anterior) during active knee flexion and extension in supine was given. Glides were given in supine lying in the direction which reduced the pain and improved the affected Range of Motion. MWM was given in 3 sets of 6 repetitions each during active knee flexion and extension. The glide force was sustained while the subject performed 6 repetitions of active full range knee flexion and extension. The technique was given in supine lying position.

$\mathrm{K}$ - T ape was applied in form of superior $\mathrm{Y}$ technique for the stabilization and support to the patella and surrounding muscles.

The kinesio Y strip tape was applied light with $25 \%$ stretch tension on medial side of knee joint and $50 \%$ stretch tension was applied on lateral side of the knee joint. I form strip tape was applied over the inferior pole of the patella to maintain the mechanical correction.
Following the intervention after forty eight hours, post intervention data was collected for muscle strength, pain, balance and coordination.

\section{Case Description 1}

A case of 59 year old female patient washaving severe pain in knee joint from past 2 years. She haddifficulty in walking and in performing ADLs. Patient was apparently asymptomatic before 2 years then suddenly she felt some pain and stiffness in right knee. She was taking physiotherapy treatment at home since 6 months. She had no past medical history of Hypertension and diabetes mellitus. The patient reported pain on NPRS as 7/10. On observation, built of the patient was endomorphic. Swelling was present on the right knee. There was no redness and any deformities. On palpation, she had tenderness of Grade 2 in lateral aspect of right knee joint. On examination MMT was done for muscle strength testing for quadriceps and hamstrings and MMT score were quadriceps $3 / 5$ and hamstring $4 / 5$ for right knee and quadriceps $4 / 5$ and hamstring 5/5 for left knee. The patients Time Up and Go score was 14 seconds, Berg Balance score was 49, WOMAC osteoarthritis index scores mostly sowed moderate to severe pain in the questionnaire.

The physiotherapy management given was 3 sets of Mulliganmobilization glides given Anterior- Posteriorly, Lateral-Medially on 1st day. Then Y-Shape kinesio taping was applied.

These showed immediate relief in pain and stabilization of knee joint. Although pain relief was short term till 48 hours of treatment. Moreover treatment showed improvement in scales we had taken for balance. The TUG score came out to be 10, Berg score went up to 52 , and NPRS was $3 / 10$.

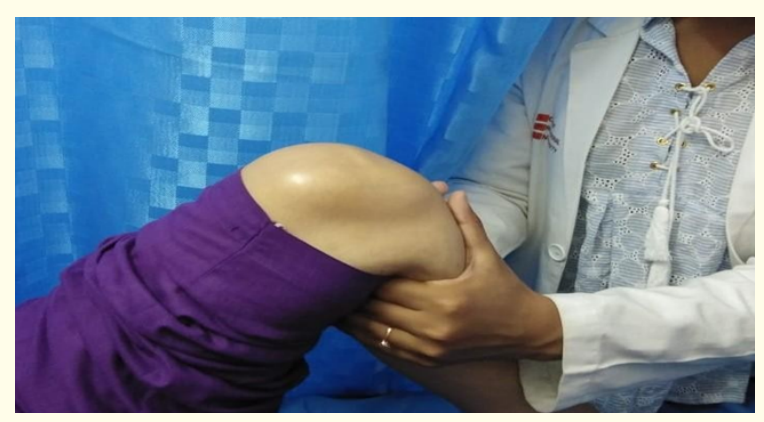

Figure 1.

\section{Case Description 2}

A 53 years old patient Harsharan Kaur, came with the complaint of bilateral knee pain(left more than right)due to which her activities of daily living were altered, this pain started almost 8 years 
ago and at that time she just used to take painkillers for the same. She is coming for regular physiotherapy treatment since 1 year to LPUopd, Phagwara. Patient had no past history of diabetes mellitus, hypertension. The patient reported pain on NPRS as 7/10.0n observation, the built of the patient was endomorphic and there was no redness or swelling nor any deformities were present. On palpation, grade 3 tenderness was found in both the medial and lateral aspects of the left knee. ON examination, MMT for hamstring was $4 / 5$ and for quadriceps was $4 / 5$ for left knee and for MMT quadriceps $4 / 5$, hamstring $4 / 5$ for right knee. Patients time up and go score came out to be 18 seconds, Berg balance score was 36 ,Womac score was showed mostly severe to extreme. Physiotherapy management was done with mobilization of 6 glides,3 sets and then $\mathrm{K}$ taping of $\mathrm{Y}$ shape was applied on her left knee.

Post treatment results-Physiotherapy intervention 3 sets of Mulligan mobilization glides given Anterio- posteriorly, posterior anteriorly. Then, Yshape kinesio taping is applied. These showed improvement in her condition, the tug test score came out to be 14 seconds and the berg balance score also improved it went up to 44 from 36.The intensity of pain as noted by Numerical Pain Rating Scale drastically reduced to 5 from 7 there was also considerable improvement in Western Ontario McMaster University Osteoarthritis index score.

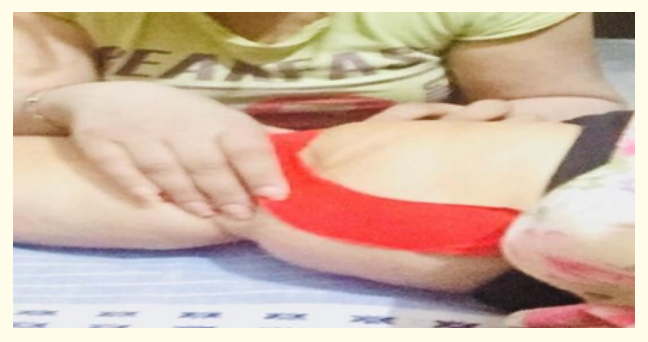

Figure 2

\section{Case Description 3}

A case of 58 year old patient described pain in both knees causing difficulty in walking and standing for prolonged time. It also disturbed her activities of daily living. Patient describes of condition being progessing since last 5 months. This was when pain started to disturb her psychologically. She has been taking regular physiotherapy treatment from LPU OPD, Phagwara since last 5 days. Patient had no past history of diabetes mellitus, hypertension. The patient reported pain on NPRS as 6/10.0n observation, the built of the patient was mesomorphic and there was no redness or swelling nor any deformities were present. On palpation, grade 2 tenderness was found in both the medial and lateral aspects of the left knee. On examination, MMT for hamstring was 3/5 and for quadriceps was 4/5 for left knee and for MMT quadriceps 4/5, hamstring $3 / 5$ for right knee. patients Time Up and Go score cam out to be 13 seconds, Berg balance score was 45, WOMAC score came out to be 29. Physiotherapy interventions given were mulligan mobilization of 6 glides, 3 sets and then $\mathrm{K}$ taping of $\mathrm{Y}$ shape was applied on her left knee.

Post Treatment-Pain reduced drastically after treatment by application of combination of kinesio taping and mulligan mobilization, the tug test score came out to be 10 seconds on post treatment day and the berg balance score also improved it went up to 53 from 43.The intensity of pain as noted by Numerical Pain Rating Scale also reduced subsequently to 5 from 7 and the Western Ontario Mcmaster University Osteoarthritis index score also showed considerable improvement decreased from 33 to 22 .

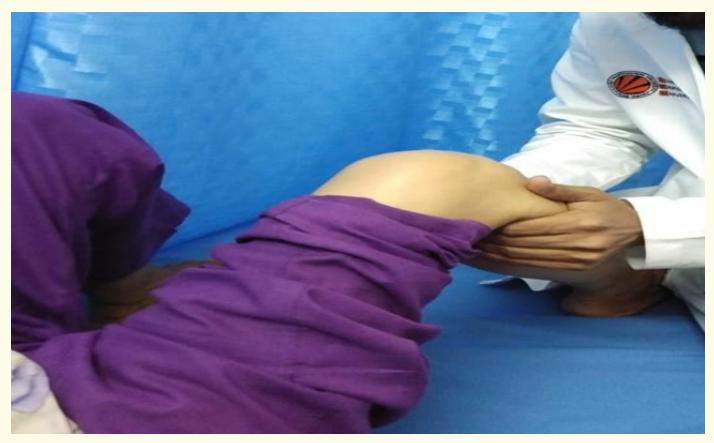

Figure 3

\section{Case Description 4}

A 57 year old female patient came with Right knee pain which is affecting her walking and exercise tolerance. Patient describes severe pain in climbing stairs. This patient has been undergoing treatment in LPU OPD since last 10 days. Patient is neither diabetic nor does she have any past history of hypertension. On NPRS scale the patient describes having a score of 8 when asked to rate from 1 to 10 with reference being given about no pain and 10 being extreme. On observation, the built of the patient was mesomorphic and there was no redness or swelling nor any deformities were present. On palpation, grade 2 tenderness was found in both the medial and lateral aspects of the left knee. On examination, MMT for hamstring was $4 / 5$ and for quadriceps was $3 / 5$ for left knee and for MMT quadriceps $4 / 5$, hamstring $4 / 5$ for right knee. The tug test score was 13 seconds prior to the treatment and the berg balance score came out to be 48.Along with these scores WOMAC score was also recorded and it came out 34 .

The patient was then treated with conventional treatment approach combining the Kinesio taping technique Y shaped with Patella stabilized and Mulligan mobilization performed in pain free movements. 
POST Treatment results- Balance and exercise tolerance significantly showed great results. The timed up and go test score went from 13 seconds to 10 seconds while the Berg balance score also went up from 48 to 54.NPRS score decreased from 8 to 6.There was considerable increase in knee stability and pain relief.

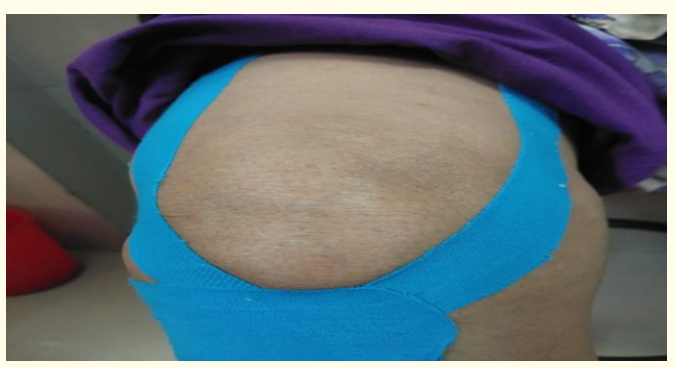

Figure 4

\section{Case Description 5}

A case of 60 years old patient described pain in left knee causing difficulty in walking. It also disturbed her activities of daily living. Patient was apparently asymptomatic five years ago but then she had a fall from height approximately 1 floor while working at a factory. That is when pain started in her knee. She has been taking regular physiotherapy treatment from LPU OPD, Phagwara since 2 years. Patient had no past history o diabetes mellitus, hypertension. The patient reported pain on NPRS as 8/10.0n observation, the built of the patient was mesomorphic and there was no redness or swelling nor any deformities were present. On palpation, grade 3 tenderness was found in both the medial and lateral aspects of the left knee. ON examination, patients time up and go score came out to be 17 seconds, Berg balance score was 39, WOMAC osteoarthritis index scores mostly showed moderate to severe pain in the questionnaire. On examination MMT was done for muscle strength testing for quadriceps and hamstrings and MMT scores were quadriceps 4/5 left knee, hamstrings $4 / 5$ left knee, 5/5 right knee for both quadriceps and hamstrings. Physiotherapy management was done with mobilization of 6 glides, 3 sets and then K taping of $y$ shape was applied on her left knee. This showed pain relief and knee stabilization immediately.

Post Treatment-Her condition improved significantly after treatment by application of combination of kinesio taping and mulligan mobilization, the tug test score came out to be 15 seconds and the berg balance score also improved it went up to 48 from 39.The intensity of pain as noted by Numerical Pain Rating Scale drastically reduced to 6 and the Western Ontario Mcmaster University Osteoarthritis index score also showed considerable improvement.

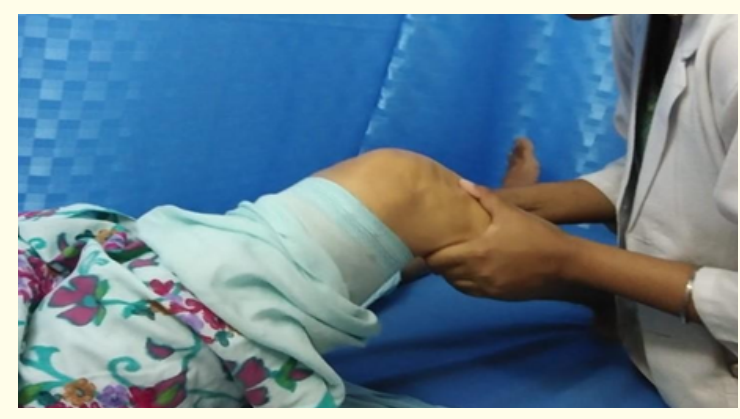

Figure 5

\section{Results}
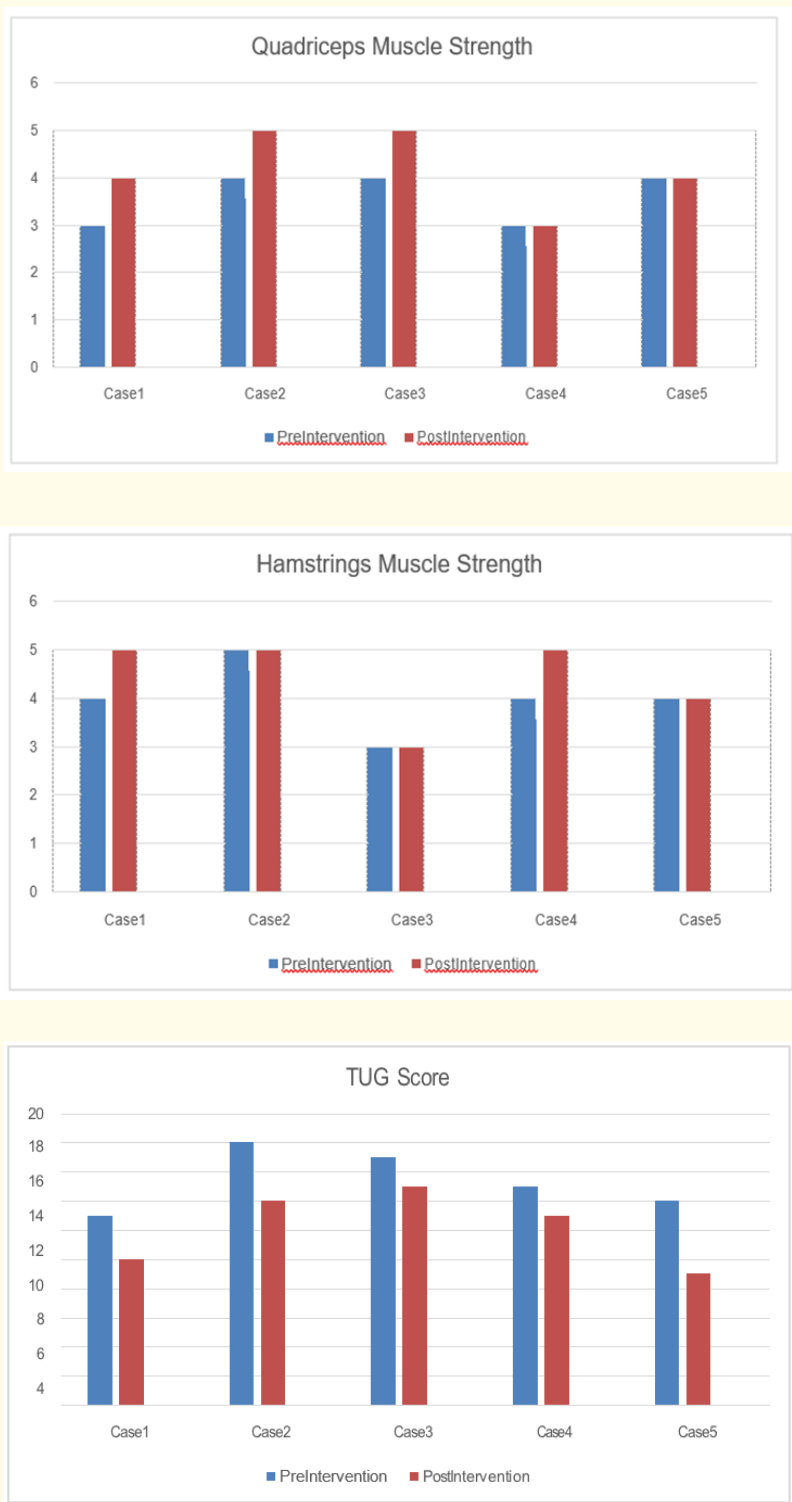

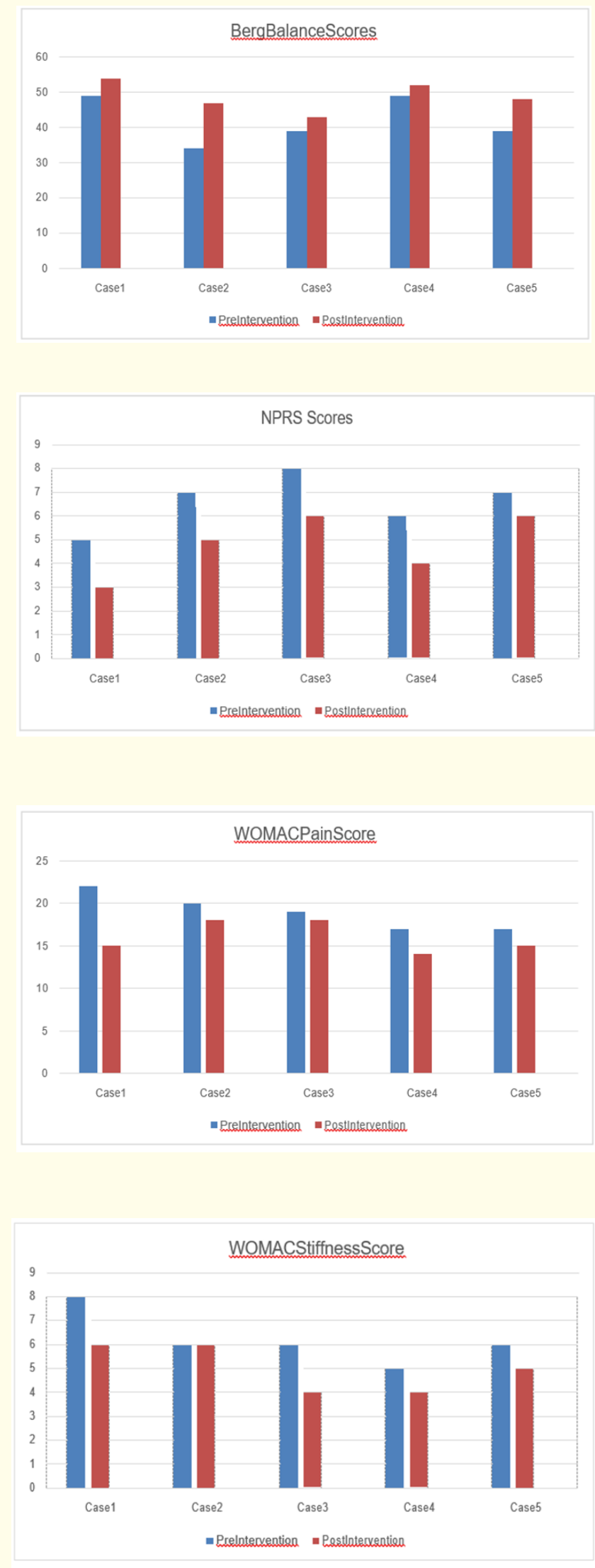

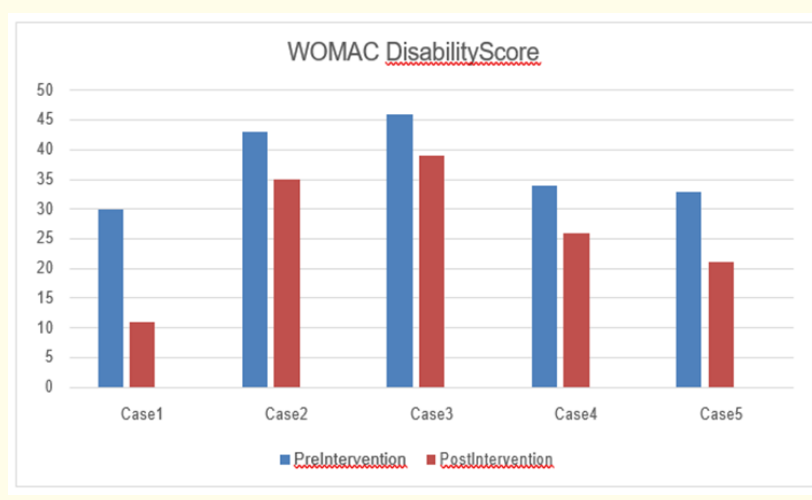

Figure 6

The study was done on 5 female subjects. Within the subjects we found that there is a significant change in means of NPRS, BERG SCORE, TUG SCORE and WOMAC score were analyzed from pre intervention to post intervention of combination therapy of Kineso taping with MWM.

Significant improvements from baseline were detected following the initial treatment. MWM is associated with immediate pain relief and improved knee function, suggesting its potential as a component of early management of knee $\mathrm{OA}$.

\section{Discussion}

In our study we found that there is a significant improvement of pain, coordination and balance and risk of fall with osteoarthritis of knee. The improvement could be because of neurophysiological mechanism of Mulligan with mobilization and biomechanical correction of the knee joint. The improvement is because of taping that allows a partial full range of motion applied muscle and the joint with different pulling force to the skin.

Comparison of pre-intervention and post-intervention in patient with knee osteoarthritis Grade 1and 2 means found that there is a significant improvement of pain and functional ability. There were no patient who responded to rotation and lateral glide (anterio-lateral and anterio-medial).

Literature prove that tape lift the skin and increases the space between the skin and muscle so it could reduce the localize pressure and will help to promote circulation leads to reduce in pain, swelling, and muscle spasm. Therapeutic K-Tape application and YPositioning of tape maintain the knee in more stable position and this reduce strain and stress on the soft tissue that improve the knee osteoarthritis symptoms. 
Literature also proves that Therapeutic Tape improves the proprioception of the joint by the normalization of muscle tone, reduction in pain, and the stimulating effect of the receptors.

We found that Mulligan movement with mobilization with combination of Therapeutic Knee Taping is an effective treatment for the management of osteoarthritic symptoms including management of pain and improving balance in particular.

Recommendation for future research: Studies are needed to find long term effect of Mulligan Mobilization with combination of K-Taping. In terms of mobilization joint partner bone alignment which means correcting the positional faults can be corrected in tibio femoral joint and patella femoral joint which may relief long term pain and functional disability in future. Although the present study was a randomized trail sub methodological limitations were present. Motion of muscles was not measured.

Furthermore, it was a sort term study and there is a need to investigate as to which of the glides were more beneficial that is AP and PA combine with K- Taping. At last it is needed to seeing in future fir how long the MWM effects last for and weather K taping wit mobilization is effective in reducing the number for total knee replacement in future [14-16].

\section{Conclusion}

The study concluded that Mulligan's Mobilization with K-Taping techniques significantly shown short term effect on improvement of pain, balance, Range of Motion and prevent the risk of fall in osteoarthritic knee patient. Therefore, use of Kineso-taping with Y- shaped is recommended for treatment of osteoarthritic knee symptom. In conclusion, Kinesio knee taping may be an effective method for short term management of pain and immediate pain relief. It can also be beneficial in management of disability in patients with knee OA. Taping may perform a role in providing sense of mechanical stability to knee. It can enhance proprioception in healthy subjects.

\section{Bibliography}

1. GUO Yinben., et al. "Progress of the Research on Wearable Triboelectric Nanogenerator". Progress in Chinese materoals (2016): 91-100.

2. Xianming He. "The Research of Flexible Triboelectricity Nanogenerator Based on PDMS Composite Film". Chongqing University (2015).

3. Wang Z L and $\mathrm{Wu}$ W. "Nano-enabled energy harvesting for self-poweredmicro/nano-systems". Angewandte Chemie International Edition 51.47 (2012): 11700-11721.
4. Huangwei. "Research on the Prospect and Investment of Wearable Device". Lanzhou University (2015).

5. Dong F L. "Research on the industry chain and trend in development of industry of wearable device". Guangdong Academy of Social Sciences (2015).

6. Shuntian FENG. "The present situation and trend of wearable equipment". Information and Communication Technology 03 (2014): 52-57.

7. Yi G., et al. "The Present Situation and Prospect of Wearable Equipment". Electronic Science and Technology (02) (2014): 238-245.

8. Wikipedia [EB/OL] (2014).

9. Feng ST. "The present situation and trend of wearable equipment". Information and Communication Technology (2014): 52-57.

10. Business Insurance. Wearable Devices' Risks Emerge 48.19 (2014).

11. Song BL. "Overview of the current situation and future development of wearable equipment". Silicon Valley (2014): 9-10.

12. Starner T. "Human-powered wearable computing". IBM Systems Journal 35. (3/4) (1996): 618-629.

13. ZL Wang. Adv. Mater. 24 (2012): 4632. b) ZL. Wang, ACS Nano (2013): 9533.

14. u X., et al. "A self-charging power unit by integration of a textile triboelectric nanogenerator and a flexible lithium-ion battery for wearable electronics". Advanced Materials 27.15 (2015): 2472-2478.

15. Z L Wang. "The principle and Potential Application of Piezoelectric Nano-generator". Physical 11 (2006): 897-903.

16. ZL Wang. "The Principle and Potential Application of Piezoelectric Nano-generator. b) Guo Y B, Zhang Q H., Li Y G, Wang H Z. "Progress of the Research on Wearable Triboelectric Nanogenerator". Materials China (2016):91-100-127

17. Zheng Z SHAO. "Study on One-Dimensional Zn O Nanostructures Based Piezoelectric Nanogenerator". National University of Défense Science and Technology (2010).

18. Zang Y., et al. Materials Horizons 2.2 (2015): 140-156.

19. Yang Y and Wang L Y. "Composite electromagnetic - triboelectric nanogenerator". Chinese Science Bulletin (2016):12681277. 
20. Xu S., et al. "Self-powered nanowire devices". Nature Nanotechnology 5 (2010): 366-373.

21. Sun Jing. "Fabrication and Performance of Triboelectric Generator Based on Biocompatible Materials". Tianjin University of Technology (2015).

22. Wu J., et al. Chemical Reviews 115.7 (2015): 2559-2595.

Volume 2 Issue 9 September 2019

(C) All rights arereserved by Md Firoj Alam., et al. 